\title{
Multimodal Training Between Agents
}

\author{
Matthias Rehm \\ Multimedia Concepts and Applications \\ Institute of Computer Science, University of Augburg \\ D-86153 Augsburg, Germany \\ rehm@multimedia-werkstatt.org
}

\begin{abstract}
In the system LOCATOR ${ }^{1}$, agents are treated as individual and autonomous subjects that are able to adapt to heterogenous user groups. Applying multimodal information from their surroundings (visual and linguistic), they acquire the necessary concepts for a successful interaction. This approach has proven successful in a domain that exhibits a remarkable variety of possible (often language-specific) structurings: the spatial domain. In this paper, the further development is described (LOCATOR ${ }^{2}$ ) that allows for agent-agent interactions such that an agent is instructed by another agent that plays the role of a teacher.
\end{abstract}

\section{Introduction}

Current literature on interface agents treats the feature of embodiment in one of two ways. Either as a matter of design or as a possibility to convey more information to the user by the use of gestures or facial expressions. But embodiment may go a step further, regarding the agent as an individual entity with specific sensory abilities and specific ways of interacting in and with its environment (e.g., [5]). In this paper, agents are introduced that learn relevant concepts through multimodal input and even trade this knowledge to other agents in their surroundings.

\section{Related Work}

The work of Cassell and colleagues on the Rea agent ([6]) is a good examples of embodiment as a means to more communications channels. Rea, short for Real-Estate Agent, is a multimodal conversational interface agent that shows users around virtual houses. An important features of this approach is the use of the agent's body to convey certain communicative functions like turn-taking or initiation. Another interesting approach combines embodiment and the modeling of emotions. André, Rist, and colleagues ([1], [7]) describe sales presentation teams that use their bodies to convey certain personality traits like introvert vs extrovert.

${ }^{1}$ The original work on LOCATOR was funded by the German Research Foundation (DFG) in the framework of the graduate program Task-Oriented Communication. 


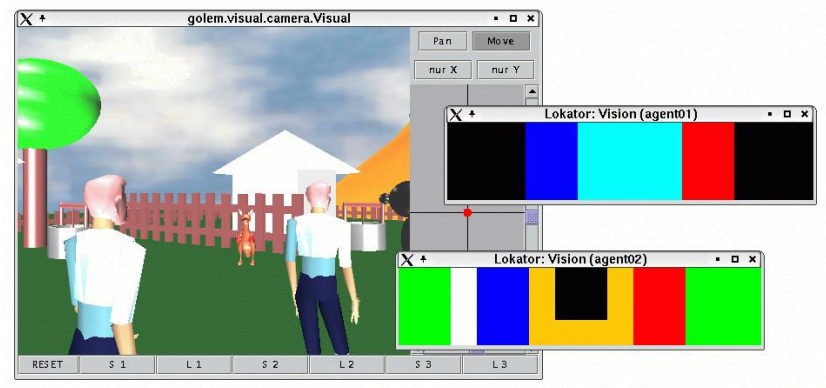

Fig. 1. A snapshot of LOCATOR ${ }^{2}$. The agent in front is the teacher who waits for the learner to catch up.

Few approaches are concerned with how an (embodied) interface learns on the basis of its perceptions. Billard and Hayes present robotic agents that learn object concepts on the basis of their sensory input and that even may teach each other labels for these concepts ([2]). The concepts are formed without taking the labels into account. Thus, they play language games similar to those described in [10]. In LOCATOR, the linguistic input is part of the concept formation process because it gives a positive example of a possible structuring of the perceivable reality.

\section{Teaching an Agent}

LOCATOR is a testbed for embodied agents that learn via multimodal input. Virtual anthropomorphic agents move around in a virtual, complex world (see Fig. 1). Different sensors allow them to receive two kinds of input during their exploration: visual and linguistic. The agents acquire individual concepts depending on their embodiment (i.e., their specific sensory equipment) and the specific situations they encounter. The linguistic input describes spatial relations between objects in one of two frames of spatial reference ([3], [8]): either in German (relative frame of reference) or in Marquesan ${ }^{2}$ (absolute frame of reference). Relative and absolute frames of reference have different logical implications concerning standpoint and orientation of the speaker. Relative frames of reference make use of reference axes that are anchored in the speaker whereas absolute frames of reference are anchored in the environment and consequently are unaffected by the spatial orientation of the speaker. Thus, it is not possible to map utterances from one into the other language without knowing the exact context in which the utterance was produced.

Steels ([9]) has proposed discrimination games to model the process of situated acquisition of object concepts. An initial feature detector exists for each possible feature. This initial detector ranges over the whole value range of the corresponding feature. Consequently, it is always activated if a value for this

\footnotetext{
${ }^{2}$ Marquesan speakers employ a directed axis sea - land (tai - uta) and an undirected cross axis $(k o)$ (see [4] for details on Marquesan).
} 


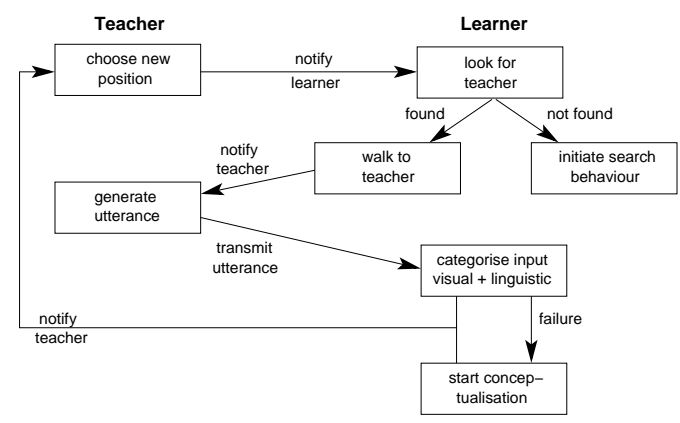

Fig. 2. Overview of the coordination behavior between teacher and learner.

feature is encountered. During the process of concept formation, the initial feature detectors are elaborated, resulting in a number of different discrimination nets, one for each perceptual feature. Each node is itself a feature detector that corresponds to parts of the value range of the given perceptual feature and is activated if a value falls into this range. In LOCATOR, this approach was modified and extended to take linguistic input into account. The pressure to modify an existing discrimination net results in Steels' approach from the assumption, that identical objects do not exist. Thus, a distinctive set of features can be determined for every object. Discrimination nets are build up only on the information supplied by the available sensors without taking linguistic input into account. In LOCATOR the linguistic input realizes a generally accepted way of structuring the spatial domain and is given as a positive example to the learner. Receiving a linguistic input triggers a categorization attempt and, if this fails, a learning step. The success of this categorization attempt is measured. The visual and linguistic input activate concepts that represent the joint meaning of the different types of input. If a single concept is activated, the categorization attempt is successful.

\section{Coordinating Teacher and Learner}

To achieve a successful communication, teacher and learner have to coordinate themselves. Because the sensory abilities of the agents are limited, this can happen only by linguistic or visually perceivable means. The coordination behavior consists of several interaction steps between the agents (Fig. 2). i.) The teacher chooses a position, which is suited for a spatial description. The autonomous exploration behavior of the agents comes into play here. Then the teacher notifies the learner that she is ready and waiting for him. ii.) Receiving this message, the learner passes it to his natural language interface to analyze it. He tries to find the teacher employing his visual sensor. If he sees the teacher, he starts walking in her direction. Otherwise he starts looking for the teacher by walking around in circles. Having reached the teacher, the learner orients himself and notifies her. iii.) When the teacher has analyzed this message, she starts generating an utterance that describes the currently visible scene. This task corresponds to a 


\begin{tabular}{|l||l|l|l|}
\hline & df & $F_{\text {succ }}$ & $F_{\text {err }}$ \\
\hline Agents & 4,89 & 0.77 & 0.55 \\
Relations & 1,89 & 0.24 & 1.08 \\
A x R & 4,89 & 0.35 & 0.81 \\
\hline \multicolumn{2}{|c|}{$p<0.05^{*}, p<0.01^{* *}$}
\end{tabular}

Categorization success

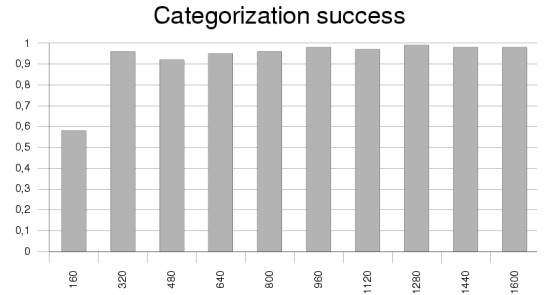

\begin{tabular}{|l||l|l|l|}
\hline & df & $F_{\text {succ }}$ & $F_{\text {err }}$ \\
\hline Agents & 9,179 & 0.15 & 0.23 \\
Relations & 1,179 & 0.02 & $6.66^{* *}$ \\
A x R & 9,179 & 0.19 & 0.73 \\
\hline \multicolumn{2}{|c|}{$p<0.05^{*}, p<0.01^{* *}$}
\end{tabular}

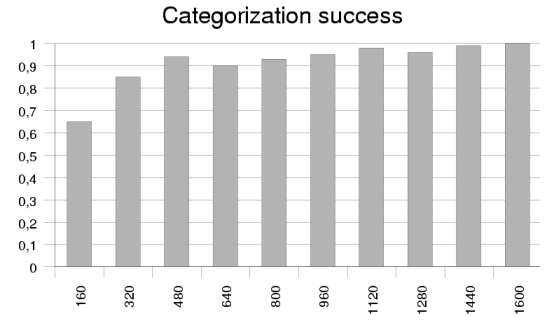

Fig. 3. Comparing results for LOCATOR (left) and LOCATOR ${ }^{2}$ (right): Analysis of covariance (above) and mean categorization success (below).

user-agent interaction where the user requests the agent to describe the scene, i.e., neither the figure nor the ground object are known. They have to be determined by the teacher. The generated utterance is transmitted to the learner. iv.) The learner tries to categorize the multimodal input. If this process fails, a learning step becomes necessary, resulting in the creation of a new concept, in the modification of an existing concept, or in initiating the modification of existing feature detectors. At last, the teacher is notified that a new round may begin.

Figure 1 shows a snapshot of the coordinating behavior of the two agents. The teacher (in front) has already reached a position to generate a spatial description and has notified the learner that she is waiting. The learner is in a convenient position as he can see the teacher. The coordination of teacher and learner as well as the instruction of the learner are based on the sensory abilities of the agents and thus depend on their specific embodiment.

\section{Results}

In a first simulation, a group of ten agents had to learn the left/right-dichotomy. They are instructed by a teacher agent that has learned this dichotomy from the user. A single agent autonomously explores its environment, i.e. it follows a random path through its environment based on local behaviors to avoid collisions with objects. From time to time the teacher describes the spatial arrangement, which the learner perceives with his visual sensor. The teacher's input triggers a categorization attempt and, if this fails, a learning step. The same kind of simulation was conducted in the original LOCATOR system which allows for a comparison of the results. Each agent is confronted with 1600 German utterances that realize the relations rechts (right) and links (left). Due to a variation across agents in the specific number of uses of each relation, an analysis of co-variance is necessary. Categorization success is measured every 160 trials as 
the mean value over the last 160 trials. Figure 3 (above) gives the results for the original system (left) and for LocATOR ${ }^{2}$ (right). Concerning categorization success $\left(\mathrm{F}_{\text {succ }}\right)$, there is not significant effect, neither for performance between agents nor for performance regarding the two relations. In $\mathrm{LOCATOR}^{2}$, a significant effect shows up for the number of false categorization attempts between the two relations $\left(F_{\text {err }}=6.66, p<0.01^{* *}\right)$. Although the agents use both concepts equally successful, they make more errors with the concept right. Because there is no significant effect between agents, a probable explanation is, that this left/right-deficiency is learned from the teacher. This remains to be shown. Figure 3 (below) shows the mean categorization success for the systems LocATOR (left) and LocATOR ${ }^{2}$ (right). At the end of the simulation, categorization success is near $100 \%$ in both cases. The mean over all trials is $93 \%$ for LocATOR and $91 \%$ for LOCATOR ${ }^{2}$. This difference between the mean performance and the performance at the end of the simulations is due to the fact, that the agents start with no concepts at all and thus will frequently make errors at the beginning.

\section{Conclusion}

Appointing an agent as teacher has proven a successful extension of the LOCATOR system. Next, more complex learning tasks will be examined, that allow for more variation in the acquired concepts and thus will also allow for individual flaws of the individual agents. The hypothesis is, that such individual flaws will make the agents more believable.

\section{References}

1. E. Andre and T. Rist. Presenting through Performing. In H. Lieberman, editor, Proceedings of IUI 2000, pages 1-8, 2000.

2. A. Billard and G. Hayes. DRAMA, a Connectionist Architecture for Control and Learning in Autonomous Robots. Adaptive Behavior, 7(1):35-63, 1999.

3. M. Bowerman and S. C. Levinson, editors. Language acquisition and conceptual development. Cambridge University Press, Cambridge, 2001.

4. G. H. Cablitz. The Acquisition of an Absolute System: Learning to talk about SPACE in Marquesan. In Proc. of the 31st SCLR Forum, 2002.

5. K. Dautenhahn. The Art of Designing Socially Intelligent Agents. Applied Artificial Intelligence, 1998.

6. J. Cassell et al. Designing embodied conversational agents. In J. Cassell et al., editor, Embodied conversational agents, pages 29-63. MIT Press, Cambridge, MA, 2000.

7. T. Rist et al. CrossTalk: An Interactive Installation with Animated Presentation Agents. In E. Andre et al., editor, Proc. of COSIGN02, pages 61-67, 2002.

8. S. C. Levinson. Frames of Reference and Molyneux's Question. In P. Bloom et al., editor, Language and Space, pages 109-169. MIT Press, Cambridge, MA, 1996.

9. L. Steels. Perceptually grounded meaning creation. In M. Tokoro, editor, Proc. of the Int. Conf. on Multi-Agent Systems, pages 338-344. AAAI Press, 1996.

10. L. Steels and F. Kaplan. Bootstrapping Grounded Word Semantics. In T. Briscoe, editor, Linguistic evolution through language acquisition. CUP, Cambridge, 1999. 